\title{
El derecho al olvido digital: una brecha entre Europa y Estados Unidos
}

\section{The right to be forgotten: a gap between Europe and United States}

\author{
Moreno Bobadilla, A. ${ }^{1}$ \\ Recibido: 10-09-2018 - Aceptado: 15-01-2019 \\ DOI: https://doi.org/10.26441/RC18.1-2019-A13
}

\begin{abstract}
RESUMEN: El derecho al olvido digital se ha convertido en tema de capital importancia, como consecuencia del impacto que Internet está teniendo sobre la vida privada de los ciudadanos. En este contexto, el presente artículo ${ }^{2}$ analiza las diferentes formas de protección de este derecho emergente en los sistemas codificados y en los sistemas del common law, para dar cuenta de las dispares realidades que se están conformando en ambos Continentes, en donde se trata de equilibrar el derecho a la información con el derecho al olvido en una era donde la memoria digital no olvida ni perdona.
\end{abstract}

Palabras clave: derecho al olvido digital; Internet; derecho a la información; derecho a la privacidad.

ABSTRACT: The right to be forgotten has become a matter of capital importance, as a consequence of the impact that the Internet is having on the privacy of citizens. In this context, this article analyzes the different forms of protection of this emerging right in codified systems and common law systems, in order to account for the different realities that are being created in both continents, where the aim is to balance the right to information with the right to be forgotten in an era where digital memory does not forget or forgive.

Keywords: right to be forgotten; Internet; right to information; right to privacy.

\section{Introducción}

Hace pocos meses se conocía la noticia relativa a la venta de datos personales de 50 millones de usuarios de la red social Facebook, a la ya desaparecida consultora británica Cambridge Analytica . Miles de usuarios de la red social más utilizada del mundo, se descargaron una aplicación, llamada "this is your digital life", y respondieron a una simple encuesta que supuestamente estaba dirigida a fines académicos. Los datos de todos estos usuarios, así como los de todos sus contactos, fueron recolectados con objeto de poder influir en sus decisiones políticas en la campaña de

1 Ángela Moreno Bobadilla es Doctora en Derecho por la Universidad Complutense de Madrid y Graduada en Derecho por la Uned. Profesora de Derecho Constitucional y Directora de Postgrados de la Universidad Andrés Bello (Chile). angmoreno.ang@gmail.com, https://orcid.org/0000-0003-4745-1901

2 Artículo realizado en el marco del Proyecto Fondecyt de Iniciación N 11180172 "Aspectos fundamentales para la configuración y el desarrollo del derecho al olvido digital en Chile". 
Donald Trump. Solamente la respondieron 270.000 personas, pero se recolectaron los datos de 50 millones.

Este caso manifiesta de forma ferviente la necesidad de aumentar la seguridad jurídica de los miles de millones de personas que diariamente usan Internet (Grimelmann, 2009; Austin, 2003). Además, esta es tan solo una de las innumerables situaciones que existen sobre vulneración de los derechos fundamentales de los usuarios en el entorno virtual (Solove, 2004).

Es precisamente en este contexto, donde el derecho al olvido digital se ha convertido en cuestión de capital importancia, debido al impacto que la Red tiene sobre la vida privada de los ciudadanos.

Hasta la creación de la Web 2.0, la memoria tradicional imperaba sobre la memoria digital, abocando al olvido de ciertos datos, de ciertas informaciones, que el mero transcurso del tiempo convertía en irrelevantes (Pérez, 2016; Tutt, 2015; Solove, 2007). "For human beings, forgetting is easy and remenbering is hard (...). Modern techonology changes this paradigm. With computers and electronic devices, remembering, rather than forgetting is increasingly the default" (Coonley, 2010: 53).

Pero en la actualidad cualquier dato pasado aparece presente tan solo tecleando en un buscador el nombre de la persona sobre la que se desea obtener cualquier tipo de información, lo que impide que los ciudadanos puedan tener una segunda oportunidad (Ayala, 2016; Mayer- Schönberger and Cukier, 2013).
Tal como sostiene Meg Leta Jones (2016: 3):

To drive home, the importance and difficulty of the issue, imagine the worst thing you have ever done, your most shameful secret. Imagine that cringe- inducing incident somehow has made its way online. When future first dates or employers or grandchildren search your name, that incident may be easily discoverable. In a connected world, a life can be ruined in a matter of minutes, and a person, frozen in time. Keeping that embarrasing secrett offline is not as easy as it once was.

Es necesario aclarar que el derecho al olvido digital tiene múltiples vertientes, en función del soporte en el que esté contenida la información objeto de controversia. Pero el siguiente artículo académico se va a centrar, principalmente, en qué ocurre con las peticiones ciudadanas de eliminación de noticias que han sido publicadas en medios de comunicación on line.

De gran relevancia es esta aclaración, ya que, en el caso de noticias o informaciones recogidas en las redes sociales $\mathrm{u}$ otros soportes virtuales, la confrontación de derechos es diferente.

Sin embargo, en estos casos en concreto, se trata de establecer si debe prevalecer el derecho a la información o el derecho a la privacidad, honra, propia imagen o protección de datos personales.

En el fondo, es la clásica confrontación de derechos que se lleva debatiendo durante siglos, pero con un nuevo añadido: las noticias aparecidas en 
Internet tienen un carácter de perpetuidad, y además no solamente son conocidas por personas que están ejerciendo su derecho a la información, sino simplemente porque se googlea el nombre de alguien sobre el que se desea saber toda la información posible (Mayer- Schönberger, 2009; Solove, 2007).

Hasta hace poco tiempo, una noticia publicada en un medio de comunicación tradicional (prensa, radio o televisión), pronto desaparecía del recuerdo de las personas. Pero en la actualidad, los datos compartidos por y en Internet pueden quedarse accesibles indefinidamente. Esto provoca que ciertas informaciones contenidas en la web, que cuando se difundieron eran de interés público y tenían carácter noticiable, con el paso del tiempo se hayan convertido en irrelevantes, y sean un constante recordatorio de unos hechos que pueden suponer una vulneración a la protección de datos personales o a la intimidad de sus protagonistas.

Por ello, el presente trabajo analiza la configuración y forma de protección del derecho al olvido digital en los principales sistemas de origen romanista del Continente europeo, así como en Estados Unidos, estandarte de los sistemas jurídicos del common law, con el objetivo prioritario de referenciar un marco claro y preciso sobre las diferencias existentes a ambos lados del Atlántico. Previamente, se hace un estudio histórico que repasa los principales hitos que sentaron las bases del derecho al olvido, antes de la era digital.

Para ello se va a realizar un análisis de la normativa y la doctrina, así como de la jurisprudencia en torno al derecho al olvido digital, y que debido a la relativa novedad de la cuestión se encuentra en pleno proceso de consolidación. En este marco, es fundamental realizar una cuidadosa comparación de los diferentes sistemas jurídicos que se han encargado de regular este derecho, atendiendo a sus principales fundamentos y motivaciones, donde el método dogmático adquiere una innegable relevancia. Lo anterior debe necesariamente realizarse desde el método analítico y comparado.

\section{El derecho al olvido previo a la era digital. La necesidad de perdón y ren- dición a ambos lados del Atlántico}

Antes de la creación de Internet, los ciudadanos, tanto europeos como americanos, ya reclamaban por tener una segunda oportunidad, o sea, por un derecho al olvido. Numerosos son los precedentes que existen sobre esta cuestión.

A continuación, se va a hacer una somera descripción de los principales hitos que conformaron, a ambos lados del Atlántico, los cimientos para la actual configuración del derecho al olvido digital.

Estados Unidos está manteniendo una posición bastante crítica respecto de este derecho. El país donde radica Silicon Valley, cuna de la tecnología mundial, encuentra que la configuración de un derecho de estas características, podría suponer una violación de la Primera Enmienda Constitucional, donde se recoge la libertad de prensa y de expresión, y además proscribe 
expresamente que el Congreso pueda adoptar cualquier ley que limite la libertad de expresión: "Congress shall make no law respecting an establishment of religion, or prohibiting the free exercise thereof; or abridging of speech, or of the press; or the right of the people peaceably to assemble, and to petition the Goverment for a redress of grievances".

No obstante, no se puede olvidar que fue precisamente en este país donde surgió uno de los principales antecedentes del derecho al olvido, es decir, el derecho a la privacidad.

Fueron Samuel Warren y Louis Brandeis, quienes, a raíz de la publicación de su célebre artículo "The right to privacy", sentaron las bases para la configuración del derecho a la privacidad, "the right to be let alone" (Warren y Brandeis, 1890).

Es este momento cuando se empieza a perfilar la idea de que la intimidad de cada persona, también es un derecho que merece ser reconocido y protegido, tesis pionera, ya que hasta la fecha era complicado entender la defensa de un derecho intangible que perteneciese a la esfera personal de los ciudadanos.

Warren y Brandeis sientan las bases de un verdadero derecho a la intimidad, mostrando al mundo una nueva concepción respecto de su significación jurídica y social, aportando, entre otras ideas, la noción de que, para resolver una posible colisión entre privacidad e información, hay que tener presente que la garantía de la intimidad no es un impedimento para que toda la información de interés público pueda ser difundida (Moreno, 2017).
El derecho a la privacidad posteriormente es desarrollado y consagrado por la teoría de los cuatro "torts" de William L. Prosser en 1960. En ella se distinguen cuatro zonas distintas de invasión: intrusión, divulgación pública de hechos privados, publicidad que falsea la imagen de una persona y la apropiación del nombre o de la imagen de una persona. De ellas, son las dos primeras, las que de forma más relevante se encargan de salvaguardar la privacidad frente a los medios de comunicación, puesto que las restantes guardan una relación más estrecha con la difamación o con la protección de la propiedad (Prosser, 1960).

Además, está la idea de Westin relacionada con la privacidad, y que hay autores que señalan que es precisamente la que está más conectada con el presente derecho al olvido (Westin, 1970). "The right to be forgotten is an idea based in a Westinian conception of privacy: that people and organizations should be permited to determine for themselves when, how, and to what extent information about them is communicated to others" (McNealy, 2012:121).

A esto hay que añadir, que fue precisamente Estados Unidos, uno de los primeros países en resolver un caso de derecho al olvido anterior a la era digital: el Caso Melvin v. Reid de 1931.

Los llamativos hechos comenzaron en 1918, cuando Gabrielle Darley, de profesión prostituta, comenzó una relación amorosa con el deportista Leonard Tropp. Tras un tiempo de noviazgo, ella le dio dinero para que le comprase un anillo de bodas, sin saber que, en realidad, él planeaba casarse con otra 
mujer a la que le regaló la mencionada sortija que había comprado con el dinero de Gabrielle.

A raíz de los hechos, Gabrielle le dispara en la calle y él muere. En el juicio, ella es declarada inocente, debido a que convenció al jurado de que el revólver se disparó accidentalmente.

Años más tarde, Adela Rogers, hija de Leonard, escribió una historia, "The Red Kimono", contando todos los hechos ocurridos y dando los nombres reales de los protagonistas. Esta fue llevada al cine en 1927.

Como consecuencia, Gabrielle demandó a Adela por invasión de su derecho a la privacidad, ya que ella había rehecho su vida y clamaba por una segunda oportunidad.

La Corte de California consideró que los hechos habían producido una violación en la privacidad de la señora Darley, y que las personas deben tener derecho a olvidar y a ser perdonadas.

Este se convirtió en uno de los casos más emblemáticos de Estados Unidos, ya que se puede considerar que es un reconocimiento judicial del derecho al olvido, del derecho a tener una segunda oportunidad, donde se pueden olvidar los hechos del pasado cuando ya no tienen relevancia para la conformación de la opinión pública del presente (Friedman, 2007).

En este caso comienzan a establecerse los orígenes del derecho al olvido, que son previos a la era digital, pero que tienen un pilar común: ciudadanos que quieren volver a recuperar su privacidad, pero siempre guardando el delicado equilibrio que es necesario mantener entre este derecho y el derecho a la información.

"After Melvin, the rehabilitive function of privacy began to dwindle, and the definition of newsworthiness began to grow" (Jones, 2016: 59).

Sin embargo, después de este caso existen otros, como Briscoe v. Reader's Digest Assoc. de 1971, en los que los tribunales consideraron que un personaje público no se vuelve a convertir en persona privada por el mero transcurso del paso del tiempo. Otros casos que guardan una estrecha relación con el anterior son Barber v. Time Inc. de 1942 o Daily Times Democrat v. Graham de 1964.

Todos ellos tienen varios elementos en común. Los protagonistas de la información son personas privadas, sobre las que los medios de comunicación han publicado diversas informaciones sin su consentimiento. Ellas piden la condena, y el olvido. Pero la jurisprudencia cambia de rumbo, y no se vuelve a fallar a favor de la privacidad y de la segunda oportunidad.

Estados Unidos se vuelve a erigir nuevamente en uno de los precursores de una materia, en la que finalmente no desarrolla el mismo grado de protección que la que se produce en el continente europeo. Por el contrario, se ha convertido en el estandarte de la defensa de la libertad de prensa y de expresión por sobre el derecho a la privacidad, donde los medios de comunicación no conocen prácticamente de límites cuando se trata de defender la Primera Enmienda Constitucional, la cual además proscribe expresamente que el 
Congreso pueda adoptar cualquier ley que limite la libertad de expresión.

En palabras de Pere Simón "la jurisprudencia norteamericana reconoce a la ciudadanía un derecho a recordar y decidir teniendo en cuenta los hechos pasados" (Simón, 2015: 113).

Por otro lado, en la Europa continental, la privacidad, así como la protección de datos personales, son derechos fundamentales en la mayoría de los textos constitucionales.

En Francia, la Commission Nationale de l'Informatique et les Libertés también reconoció de forma expresa la existencia del derecho al olvido, con una amplia interpretación de lo que comprende el poder solicitar una segunda oportunidad.

Fue precisamente en este país, donde ya en el año 1965 se dicta una pionera sentencia que comienza a reconocer el derecho de los ciudadanos a tener una segunda oportunidad. El Tribunal de Gran Instancia de Sena, en sentencia dictada el 4 octubre del mencionado año, resolvió una demanda de una de las amantes del famoso asesino en serie Henri Landru, por haber sido representada en una película después de haber transcurrido muchos años desde la relación sentimental que mantuvo con el homicida. A pesar de que finalmente el órgano jurisdiccional francés rechaza la demanda, ya que la actora había publicitado su relación con el señor Landru, se comienza a hablar del "droit a l'oublie”, sembrándose los orígenes europeos del derecho al olvido previo a la era digital.

\section{La era digital del derecho al olvido. Una brecha entre ambos Continentes}

Antes de comenzar a analizar las disimilitudes en la construcción del derecho al olvido digital en Europa y en Estados Unidos, se van a explicar las principales diferencias que existen entre ambos sistemas jurídicos, entre los sistemas de origen romanista, también denominados sistemas civilistas o codificados (propios de los países de la Europa Continental, así como de Sudamérica), y los sistemas del common law (característicos de Estados Unidos, Inglaterra y Gales, Irlanda del Norte, parte de Canadá, Australia y Nueva Zelanda).

El primero de ellos está basado principalmente en el desarrollo legislativo, mientras que el segundo es un Derecho de creación judicial, es decir, son normas jurídicas que surgen de las sentencias de jueces y tribunales. Por eso se denomina Derecho de elaboración judicial. Esto significa, que las decisiones adoptadas por los tribunales en la resolución de los casos, se introducen dentro del ordenamiento jurídico por la aplicación de la doctrina del precedente (Cuñado de Castro y Gámez, 2017).

De aquí se deriva la principal diferencia entre ambos sistemas jurídicos, ya que en los sistemas codificados la jurisprudencia es una fuente secundaria del derecho, mientras que en los sistemas del common law es principal.

Además, otra importante asimetría, más centrada ya en el objeto de la presente investigación, es la preeminencia que se otorga a la libertad de prensa frente a los derechos de la personalidad 
en los sistemas del common law, especialmente en Estados Unidos como consecuencia de la fuerza de la Primera Enmienda Constitucional, tal como ya se ha señalado anteriormente (Saldaña, 2011).

En este punto es importante tener en cuenta las grandes diferencias que existen en esta materia en ambos continentes. Hay autores que defienden la idea de que en Estados Unidos no se debería imponer la configuración de un derecho al olvido digital en los mismos términos que en Europa, porque hay que analizar las particularidades sociales y culturales que presenta cada uno de los sistemas jurídicos, para poder comprender en profundidad las dos visiones antagónicas que existen en la materia (Jones, 2016; Werro, 2009; Whitman, 2004).

En palabras de Meg Leta Jones (2016: 28-29):

An important difference between European and American information policy to keep in minds is the default for sharing. The default in the U.S. generally permits the collection and transfer of personal information and prevents abuse through self- regulation and market forces. National systems within Europe operate under comprehensive regimes that protect personal information across both the public and private sectors and are enforced by specialized data- protection agencies. The estructure, resources, regulatory tools, and enforcement tactics of data- protection authorities, as well as their place within the national regulatory system, vary across na- tions, but the default is generally not open sharing.

Una idea común que defienden los autores recientemente señalados, es que es fundamental que se respete la identidad cultural de cada país. "The two Western cultures seem on irreconcilable paths when it comes to the recognition and enforcement of a right to be forgotten" (Werro, 2009: 286). Para esto hay que tener presente la forma en que se ha desarrollado el concepto de privacidad dentro de cada una de las sociedades (De Beats, 2016; Whitman, 2004).

Un claro ejemplo se manifiesta en el hecho de que en Estados Unidos la gente habla públicamente respecto del salario que percibe, mientras que en Europa es una cuestión que socialmente no está aceptada. Otro ejemplo se encuentra en el ámbito de los antecedentes penales. Mientras que en el Viejo Continente estos no son accesibles para todas las personas, en el país anglosajón estos son públicos, cuestión que está insertada y validada en la sociedad.

En palabras de Whitman: "We are in the midst of significant privacy conflicts between the United States and the countries of Western Europe-conflicts that reflect unmistakable differences in sensibilities about what ought to be kept "private"” (Whitman, 2004:1155).

Esta brecha, que será analizada a continuación, ha sido, muy acertadamente resumida por Werro: "One way to look at this divide is in terms of the cultural loci of trust: the Europeans trust in the government and distrust the market, while Americans take precisely the opposite view" (Werro, 2009: 299). 
Pero, a pesar de que este argumento es totalmente defendible desde la perspectiva de que cuando se configura un derecho siempre hay que tener presente las peculiaridades de cada comunidad (Jones, 2016; Friedman, 2007), es cierto que, tal como se va a comprobar a continuación, el grado de protección del derecho al olvido digital difiere notablemente entre Europa y Estados Unidos, aumentando la brecha que existe entre ambos Continentes respecto de cómo se resuelven las confrontaciones entre el derecho fundamental a la información y el emergente derecho al olvido digital.

\subsection{El caso particular de Estados} Unidos. El interés público de la información como concepto central de la discusión

En 2010, Harvey Purtz solicitó en el Estado de California el reconocimiento del derecho al olvido digital para su hijo, recientemente fallecido. El joven, estudiante de la Universidad de Berkeley, había sido noticia en el periódico Daily Californian en 2007, como consecuencia de unos disturbios que había protagonizado en un local de striptease en San Francisco.

Como consecuencia, fue expulsado del equipo de fútbol de la Universidad. Posteriormente, falleció en un accidente. El padre del joven, solicitó la desindexación de esta noticia, ya que debido al fatal desenlace de los acontecimientos, esta ya no era relevante ni tenía interés público, pero suponía un disturbio para la familia.

El editor del diario se negó, alegando que la información publicada era de interés público.

La Corte dio la razón al medio de comunicación, y la noticia sigue apareciendo en Internet.

En este punto, cabe preguntarse si es relevante saber que este joven fuese expulsado del equipo de fútbol como consecuencia del incidente que protagonizó, o si por el contrario, dicha información, debido al paso del tiempo, carece ya de dicho interés, y se ha convertido en mero morbo y sensacionalismo.

Pero lo cierto, es que este tipo de peticiones son vistas en Estados Unidos como una limitación a la Primera Enmienda Constitucional.

La defensa de la libertad de prensa es el principal argumento de los detractores de la configuración del derecho al olvido digital dentro del país norteamericano, donde numerosos autores se muestran en contra de que la Section 230 Communication Decency Act pueda exigir a los diferentes medios de comunicación la eliminación de ciertas informaciones.

No obstante, no se deben visualizar como dos derechos antagónicos, porque tal como defienden algunos autores, la privacidad, y por lo tanto, las nuevas formas de su protección dentro de Internet, no son el enemigo de la libertad de expresión (Bernal, 2011; Mills, 2008).

Otro llamativo caso se produjo en 2003. Katie, una adolescente de 18 años de edad, vivía en un pequeño pueblo a dos horas de la ciudad de Denver, ubicado en el Estado de Colorado. El 4 de 
julio de dicho año, Kobe Bryant fue acusado de un delito de violación, del que posteriormente fue absuelto.

Pero durante los meses posteriores al incidente, varias páginas web identificaron, de forma errónea, a Katie como presunta víctima de dicho delito, incluyendo incluso la fotografía de la joven.

Como consecuencia de la falsa información, y de vivir en una comunidad pequeña, la madre de Katie solicitó que se eliminase dicha información, alegando que:

I was really upset by the whole situation (...). It's hard knowing that when people think about Kobe's accuser, I'm the face thay everyone thinks of (...). I feel violated. I want it to be known that these pictures aren't of the right girl, and I want them removed (Jennifer 8. Lee, Net Users Try to Elude the Google Grasp, NY Times, July 25, 2002).

Desafortunadamente para Katie, no todos los sitios web eliminaron la información, alegando algunos de ellos, que en la actualidad ya no existe la privacidad. Debido a la falta de mecanismos legales que existen en esta materia en Estados Unidos, Katie tuvo que padecer esta situación, que tal vez pueda seguir estigmatizándola para el resto de su vida.

Este podría haber sido un buen punto de partida para comenzar a configurar un derecho al olvido digital dentro este país, teniendo en cuenta que el elemento central de esta discusión podría ser el interés público de la información, sumado al hecho de la falsedad de la misma.

El interés público podría convertirse en la excepción para poder acoger las peticiones relacionadas con the right to be forgotten (Koops, 2012; Coonley, 2010). Además, no se puede defender bajo este prisma las informaciones que estén basadas en rumores y hayan sido publicadas con imprudencia, ya que en estos casos no existe dicho interés (McNealy, 2012).

Pero en Estados Unidos, a diferencia de Europa, el interés público de la información no desaparece con el paso del tiempo, por lo que las historias que sean verídicas siempre estarán protegidas por dicho concepto.

Es doctrina mayoritaria la tesis de que la configuración de un derecho al olvido digital en Estados Unidos puede estar en contradicción con la tradicional jurisprudencia de la Corte Suprema relativa a la privacidad. Esta ha manifestado en reiteradas ocasiones que una información que es pública, nunca más puede volver a ser privada, y una persona que se ha convertido en personaje público, por cualquier motivo, no puede volver a solicitar un nivel de protección de su privacidad que sea comparable al de las personas privadas (Jones, 2016; McNealy, 2012). En palabras de Bennett "in a series of opinions, the US Supreme Court held that newsworthy, true stories are protected by freedom of the press, although they may conceivably cause embarrassment or other harm to the stories subjects" (Bennett, 2012: 170).

Este argumento sería realmente rebatible, ya que el derecho al olvido digital no defiende que la información se vuelva privada, sino que esta no sea accesible cada vez que se teclea el nombre de la persona que protagonizó la infor- 
mación en un buscador. Por lo tanto, esta seguirá existiendo cada vez que alguien quiera ejercer su derecho a investigar, ya que estará accesible en las hemerotecas de los diarios digitales. Esta es precisamente la diferenciación entre the right to be forgotten y the right to oblivion (Youm y Park, 2016; Balkin, 2016).

Además, no se trata de que las solicitudes siempre sean acogidas, sino que el organismo encargado en cada país, será el que tenga que determinar la pertinencia de esta petición al caso concreto. Por ejemplo, tendrá que tener en cuenta si se trata de una persona pública o privada, y en el segundo de los supuestos, habrá que valorar si el elemento temporal ha hecho decaer o incluso desaparecer el interés público de dicha información.

Pero el problema en este punto se presenta en que en Estados Unidos, a diferencia de en Europa, los motores de búsqueda no son responsables de la información que almacenan, y en consecuencia las peticiones solamente pueden estar dirigidas a la eliminación de la información de la fuente de origen. Y en este caso, sí que se puede producir una afectación de la libertad de prensa.

3.2. El arduo debate en el país que se ha convertido en la cuna de la tecnología mundial. Las diferentes posturas doctrinales y sociales de los estadounidenses

En realidad, la cuestión del derecho al olvido no es del todo ajena para Estados Unidos, desde que en enero de 2015 se publicara en California una ley,
California Senate Bill 568 2013, que permite a todos los menores de 18 años borrar (que no desindexar, sino borrar de forma permanente) toda la información que ellos mismos hayan subido a las redes sociales. Es decir, se está reconociendo the right to erause para un colectivo en concreto.

Pero esta situación, al menos hasta la fecha, todavía no es extrapolable al ámbito de los medios de comunicación on line.

En este punto, la doctrina estadounidense se encuentra bastante dividida. Una parte de la misma, es partidaria de la configuración del derecho al olvido digital dentro del país, con objeto de que los ciudadanos puedan tener derecho al perdón y a la privacidad virtual (Jones, 2016; Koops, 2012; Bernal, 2011; Solove, 2007). Y es que, tal como dice Cook, "the storage of personal information on the Internet has virtually destroyed our ability to keep even the most basic personal information private" (Cook, 2015: 123).

También Coonley es partidario de esta cuestión, pero siempre y cuando, se trate de información personal, dejando fuera la posibilidad de la información aparecida en los medios de comunicación. Además, se muestra a favor de que este derecho se construya en íntima relación con las property laws.

Esta postura es defendida por un amplio sector académico, que considera que el derecho al olvido digital pertenece a la esfera de los derechos de propiedad intelectual (Jones, 2016). En este punto, queda patente la visión económica que tienen sobre este derecho, 
debido a que en el país los datos son vistos como una oportunidad de negocios, no como un aspecto de la vida privada de los ciudadanos que es necesario proteger.

No obstante, sí que se han producido casos de eliminación de la información del universo virtual, que se encarga de analizar Section 512 del DMCA, como consecuencia de que se estaban vulnerando las leyes relativas al copyright.

Incluso se ha producido la creación de empresas del sector privado que se dedican a "limpiar" el historial virtual de las personas, como es el caso de Reputation.com, TrueRep.com y IntegrityDefender.com. Estas empresas generan una gran cantidad de contenido positivo sobre sus clientes, para que los resultados negativos queden escondidos en páginas posteriores de los diferentes buscadores. Esto constituye un claro ejemplo de cómo se está produciendo la comercialización de una cuestión tan sensible, como la que nos ocupa en esta investigación, ignorando que la privacidad es un derecho fundamental.

En el lado opuesto, se encuentra el sector doctrinal que se ha posicionado firmemente en contra de este derecho emergente (Bennett, 2012; Rosen, 2012; Werro, 2009).

Rosen es el principal abanderado de esta postura, para el cual "the biggest threat to free speech on the Internet in the coming decade" (Rosen, 2012: 88).
Además, argumenta que esto puede derivar en censura: "This could transform Google, for example, into a censorin- chief for the European Union, rather than a neutral platform" (Rosen, 2012: 92).

¿Será concordante la opinión de la doctrina con la de la sociedad? Para responder a esta pregunta hay que saber cuál es la visión de los ciudadanos estadounidenses respecto de este derecho. En una encuesta ${ }^{3}$ realizada a 81 estudiantes de la Universidad de Florida, a estudiantes comprendidos entre los 18 y los 24 años, el 100\% respondió que usaba a diario Internet, de los que el $60 \%$ respondieron que mayoritariamente lo utilizan para consultar las redes sociales, y solamente un $1 \%$ para leer los medios de comunicación on line. Respecto de los usos que hacen dentro del universo virtual, el $99 \%$ afirma que siempre usa Google como motor de búsqueda.

En este punto de partida, es sugerente el hecho de que a pesar de que al $50 \%$ le gustaría eliminar algún contenido que aparece asociado a su nombre, solamente el $32 \%$ considera que es necesario que se configure un derecho al olvido digital dentro de Estados Unidos.

Este dato resulta llamativo, ya que la mitad de los estudiantes encuestados quisieran eliminar algún tipo de contenido, debido a que son conscientes de que en un futuro pueden tener problemas respecto de esta cuestión, pero, aun así, no todos ellos están a favor de

3 Encuesta realizada durante la estancia de investigación postdoctoral que la autora desarrolló en la Universidad de Florida en 2018. 
que se regule, bajo el argumento de que la sobrerregulación puede suponer una merma de la libertad de los ciudadanos.

\subsection{La Europa continental. Un lugar seguro para la vida privada de los ciudadanos}

En contraposición, en los principales sistemas de origen romanista, el derecho al olvido digital ha cobrado especial importancia, siendo solicitado por algunos autores que incluso sea considerado como un derecho fundamental. Xanthoulis defiende que "should be conceptualised as a human right and more specifically as an expression of the broader right to privacy" (Xanthoulis, 2013: 84).

Es importante mencionar los hechos ocurridos en torno a la Sentencia del Caso Costeja, la cual marcó un punto de inflexión respecto al tema del derecho al olvido digital en Europa, debido a los criterios que asentó respecto del papel y responsabilidad de los motores de búsqueda.

El señor Costeja, reputado empresario catalán, había sido protagonista de una noticia en el periódico español de La Vanguardia porque figuraba en un anuncio de una subasta de inmuebles relacionada con un embargo de deudas a la Seguridad Social. Estos hechos habían tenido lugar mientras desarrollaba sus actividades empresariales, en las que dejó de pagar las cuotas a la Seguridad Social, que tuvieron como consecuencia el mencionado embargo.

El hecho de que cada vez que se introducía su nombre y apellidos en un buscador apareciese la mencionada in- formación, le impedían que pudiera volver a tener una segunda oportunidad, ya que argumentaba que la noticia ya no tenía interés público en la actualidad. Además, no se pedía que se eliminase la información en el periódico, sino solamente que esta no fuese accesible por el mero hecho de escribir su nombre.

La cuestión prejudicial fue planteada desde España en una impugnación que Google, y su filial en dicho país, habían entablado contra una resolución de la Agencia Española de Protección de Datos que amparaba en parte la denuncia de don Mario Costeja González.

El litigio se basaba en saber si Google estaba obligada a borrar de Internet todos los datos referidos al mencionado embargo, y que se recuperaban a través del motor de búsqueda. Dicha información pertenecía al pasado de la vida privada del ciudadano.

El Tribunal determinó que los ciudadanos pueden solicitar la eliminación de datos personales contenidos en la Red, cuando su tratamiento sea ilegítimo, o sea, que no sea adecuado, pertinente o excesivo en relación con los fines y el tiempo transcurrido. Por lo tanto, se pronunció a favor del ciudadano, exigiendo la desindexación de la información tanto de Google Inc como de Google Spain (Rallo, 2014).

No obstante, la información sigue accesible en el universo virtual cuando esta se busca a través de parámetros diferentes del nombre del señor Costeja, no implicando que la información quede suprimida de la fuente original. La única consecuencia práctica, es que el 
dato (o información no deseada) quede desvinculado del nombre concreto cuando se realiza una búsqueda.

Esto para guardar un equilibrio entre el derecho al olvido y el derecho a la información, tal como manifiesta el Tribunal de Luxemburgo (también conocido por las siglas de TJUE: Tribunal de Justicia de la Unión Europea) en la sentencia aludida.

En ella se establece que el tratamiento de datos que realizan los motores de búsqueda, debe considerarse sometida a las normas que sobre protección de datos rigen en la Unión Europea. Esto implica que se reconozca a las personas el derecho a solicitar que los enlaces a sus datos personales no figuren en los resultados de una búsqueda en Internet realizada por su nombre. Se reconoce, pues, el derecho al olvido digital.

O sea, que la información podrá ser eliminada si "es inadecuada, no es pertinente, o ya no lo es, o es excesiva en relación con los fines del tratamiento en cuestión realizado por el motor de búsqueda" (parágrafo 94). Pero, además, esto tampoco será siempre así, porque si el sujeto fuera persona pública o la información de interés público, el derecho a la información prevalecería sobre el derecho al olvido.

No obstante, hay que advertir que esta sentencia se refiere tan solo a los resultados obtenidos en motores de búsqueda tras teclear el nombre de una persona determinada. No implica, por tanto, que la página donde aparece el dato desaparezca ni que la información quede suprimida de la fuente original. La única consecuencia práctica, por tanto, es que el dato (o información no deseada) queda desvinculado del nombre concreto cuando se realiza una búsqueda, pero nada más.

Tras esta sentencia, el derecho al olvido ha adquirido en Europa una mayor relevancia jurídica, y ha terminado por formalizarse su reconocimiento como tal en el Reglamento (UE) 2016/679 del Parlamento Europeo y del Consejo, de 27 de abril de 2016, relativo a la protección de las personas físicas en lo que respecta al tratamiento de datos personales y a la libre circulación de estos, que es aplicable desde el 25 de mayo de 2018, y en cuyo artículo 17 se recoge de forma expresa el derecho al olvido como un derecho de cancelación (Moreno y Serrano, 2017).

Pero además del reconocimiento por parte de la Unión Europea, los diferentes Estados parte han procedido a adaptar, de diferentes formas, sus propios ordenamientos jurídicos en esta materia. A continuación, se va a hacer referencia a los casos de Alemania, Francia, Italia y España, debido a que son los cuatro países europeos donde se ha producido un mayor desarrollo en esta cuestión.

En el supuesto alemán el derecho al olvido digital ha entrado a formar parte del ordenamiento jurídico germánico mediante la misma fórmula que se recogió el derecho a la vida privada, es decir, a partir del libre desarrollo de la personalidad establecido en el artículo 2.1 de su Ley Fundamental. Esta fórmula es la defendida por varios autores para que se incorpore así a otros ordenamientos jurídicos (Simón, 2015). 
Con ello se evita delimitar el complejo y polisémico contenido que se debe proteger con la configuración de este derecho, porque en función de la situación y del soporte donde esté contenida la información que se desea eliminar o desindexar, se está hablando de privacidad, de honra, de propia imagen o de datos personales, todos ellos derechos fundamentales recogidos en la mayoría de las Constituciones de los sistemas de raíces continentales. $Y$ es que, no se puede configurar de manera genérica un derecho al olvido digital que englobe cualquier situación (Muñoz, 2015; Álvarez, 2015; Touriño, 2014). En palabras de Meg Leta Jones (2016: 98).

The right to be forgotten could be applied in an absurd number of circumstances and cannot apply to all information related to all individuals in all situations, no matter in what jurisdiction the right is established. Possible applications of the right illustrate the very different circumstances that the right to be forgotten attempts to take on and supports an argument for conceptual separation.

Por su parte, es importante resaltar los casos de España e Italia, en donde la Agencia Española de Protección de Datos Personales (a partir de ahora AEPD) y el Garante per la Protezioni dei Dati Personali respectivamente, llevan más de una década siendo los órganos de carácter administrativo encargados de velar por la protección de los datos de los ciudadanos en internet (Klingenberg, 2016).

Es de resaltar la labor que está desarrollando en esta materia la AEPD, de- bido a que lleva una gran cantidad de causas relacionadas con temas de derecho al olvido digital.

Incluso se puede encontrar dentro de su página web una clara guía en donde se explica, en un lenguaje fácil y accesible, cómo se debe proceder para realizar una petición en materia de olvido, con objeto de que el conjunto de la sociedad pueda ser partícipe de esta cuestión.

Este organismo de carácter administrativo es el encargado de decidir en cada uno de los casos la pertinencia respecto a si se debe estimar la petición de desindexación de la información o no. $\mathrm{Y}$ en caso de que el ciudadano no se muestre de acuerdo, puede acudir a la vía judicial para que los respectivos tribunales decidan sobre esta cuestión.

En este caso, debido al ámbito jurídico protegible del derecho al olvido digital dentro de los países de la Unión Europea, es una forma pertinente y eficaz de materializar este derecho. Es cierto que desde el punto de vista práctico de su protección, los mecanismos que se están llevando a cabo son bastante eficaces.

La AEPD se ha manifestado expresando que ningún ciudadano que sea personaje público, ni que sea objeto de hecho noticiable de relevancia pública, debe resignarse a que sus datos personales circulen por la red, siendo el responsable el motor de búsqueda, que deberá encontrar medios para que la información no vuelva a aparecer en el futuro (Guasch, 2015; Simón, 2015; Rallo, 2014; Hernández, 2013).

Esta agencia española se ha convertido en la encargada de recibir 
innumerables peticiones en donde se estudia la pertinencia o no de borrar datos que fueron almacenados sin la autorización de sus titulares en la mayoría de las ocasiones.

Desde el año 2007, cuando se produce su primera resolución, España ha sido uno de los países pioneros en ejercer este derecho con objeto de evitar la difusión universal y pertinente de los datos de los ciudadanos en Internet. En palabras de Pere Simón (2015: 217):

Los criterios utilizados integran una doctrina equilibrada, idónea y necesaria que se ha construido lenta pero progresivamente desde 2007 en base a principios racionales y de respeto hacia personas que ya han superado una fase concreta de su vida y que, en cualquier caso, no deben tener la carga de soportar, indefinidamente en Internet, la persecución de los sucesos embarazosos del pasado.

Por lo tanto, basa sus decisiones en el ya anunciado criterio del interés público de la información, así como en si esa relevancia pública sigue siendo actual. Además, tiene en cuenta si existe una finalidad legítima que justifique el tratamiento en origen y el tratamiento posterior de los datos por parte del buscador.

Pero todo ello con el claro criterio, de que en caso de que la petición sea aceptada, se debe producir solamente la desindexación de la información del motor de búsqueda, el cual es responsable del tratamiento de esos datos personales, pero nunca se debe producir la eliminación de la información de la fuente de origen.
Además, este es un tema que seguirá avanzando en gran medida en España, ya que actualmente se encuentra en tramitación en el Congreso de los Diputados un Proyecto de Ley sobre protección de datos personales y garantía de los derechos digitales y que será aprobado en los próximos meses, y cuyo objetivo es garantizar un óptimo cumplimiento de la nueva legislación europea que hay sobre la materia.

Por su parte, en Francia, la Commission Nationale de l'Informatique et les Libertés también reconoció de forma expresa la existencia del derecho al olvido, con una amplia interpretación de lo que comprende el poder solicitar una segunda oportunidad.

\section{Conclusiones}

En este punto hay una cuestión fundamental que está marcando la diferencia entre los sistemas codificados y el common law. En primer lugar, las diferencias culturales entre ambos sistemas jurídicos, provocan diferentes percepciones tanto sociales, como políticas y jurídicas respecto de esta cuestión. Y es que, mientras que los europeos confían en la regulación de los gobiernos, los norteamericanos lo hacen en la libertad del mercado.

Esto ha provocado, que en la Europa continental se haya producido un reconocimiento expreso del derecho al olvido digital. Además, al haberse establecido la jurisprudencia de que los motores de búsqueda son responsables del tratamiento de los datos personales, la información susceptible de olvido simplemente se puede desindexar, no siendo 
necesaria su desaparición de la fuente de origen, y por lo tanto, no quedando afectado el derecho a investigar ni el derecho a la información.

Sin embargo, en Estados Unidos no se concibe esta cuestión, porque los motores de búsqueda no ostentan ningún tipo de responsabilidad sobre la información que aparece en sus resultados de búsqueda.

Ahora bien, es importante que en ambos continentes se tenga en cuenta, tal como ha quedado mencionado a lo largo del artículo, las particularidades que se presentan en cada petición relacionada con el derecho al olvido, el cual no se puede aceptar siempre y en cualquier tipo de situación.

$\mathrm{Y}$ es que, a pesar de estas diferencias culturales entre ambos sistemas jurídicos, en Estados Unidos, tal vez debería haber una reflexión más profunda respecto de un posible reconocimiento en ciertas situaciones del derecho al olvido digital, que pudiese decidir la 230 Section Act de Telecomunicaciones, porque las consecuencias que están teniendo que padecer muchos ciudadanos son catastróficas.

Si debido a su tradición jurídica no están dispuestos a hacerlo en ningún caso, es porque la nueva forma de privacidad que se está demandando por parte de los ciudadanos no está siendo protegida de una forma adecuada.

En definitiva, la forma en la que se está configurando el derecho al olvido en ambos sistemas jurídicos incide en la idea de que en Europa es fundamental el respeto y la dignidad, mientras que en Estados Unidos es esencial la libertad, especialmente la libertad contra el Estado. Esto, en suma, se trata de dos nociones diferentes basadas en los mismos cimientos, que son la privacidad de los ciudadanos que conforman cada una de las diferentes sociedades.

\section{Bibliografía}

Álvarez, M. (2015). Derecho al olvido en internet: el nuevo paradigma de la privacidad en la era digital. Madrid: Reus.

Anguita, P. (2016). Acciones de protección contra Google. Análisis del llamado derecho al olvido en buscadores, redes sociales y medios de comunicación. Santiago: Librotecnia.

Austin, L. (2003). Privacy and the question of technology. Law and and Philosophy, 22, 119- 166.

Ayala, T. (2016). Memoria versus olvido: La paradoja de Internet. UNIVERSUM, 31 (1), 31- 44.

Balkin, J. M. (2016). Information fiduciaries and the first amendment. University of CaliforniaDavis Law Review, 49 (4),1128-1234.

Bennett, S. C. (2012). The "Right to be forgotten": reconciling EU and US perspectives. Berkely Journal of International Law, 30 (1), 161- 195.

Bernal, P. (2011). A Right to Delete? European Journal of Law and Technology, 2 (2), 1-18.

Cook, L. (2015). The right to be forgotten: a step in the right direction for cyberspace law and policy, Journal of Law, Technology and the Internet, 6, 121- 132. 
Coonley, C. (2010). The right to delete. AAAI Spring Symposium, Series, 54.

Corral, H. (2017). El derecho al olvido en internet: antecedentes y bases para su configuración jurídica. Revista Jurídica Digital UANDES, 1, 43- 66.

Cotino, L. (2018). Google y el derecho al olvido en Europa. Algunos "olvidos" y otras tendencias negativas respectos de las libertades informativas en internet. En Labrador, M. J. y Carter, E. (coords.), Google: derecho al olvido y desafíos éticos en el escenario mediático digital (pp. 129- 168). Santiago de Chile: Ril Editores.

Cuñado de Castro, F. y Gámez, Ruth (2017). Introducción al Common Law. Navarra: Thomson Reuters.

De Beats, A. (2016). A historian's view on the right to be forgotten”. International Review of Law Computers and Technology, 30 (1-2), 57- 66.

Fernández, L. A. (2016): El nuevo reglamento europeo de protección de datos. Foro Nueva Época, 19 (1), 395- 411.

Friedman, L. M. (2007). Guarding life's dark secrets. Legal and social controls over reputation, propriety, and privacy. Standford: Standford University Press.

Grimmelmann, J. (2009). Saving Facebook. Iowa Law Review, 94, 1137- 1206.

Guasch, V. (2015). El derecho al olvido en internet. Revista de Derecho UNED, 16, 989- 1005.

Hernández, M. (2013). Derecho al olvido en internet como nuevo derecho fundamental en la sociedad de la información. Perspectiva constitucional española y europea. Quid Iuris, 21,115- 148 .

Jones, M. L. (2016). Crtl Z The right to be forgotten (Nueva York, NYU Press).

Klingenberg, A. M. (2016). Catches to the right to be forgotten, looking from an administrative law perspective to data processing by public authorities. International Review of Law, Computers and Technology, 30 (1-2), 67- 75.

Koops, B. (2012). Forgetting Footprints, Shunning Shadows. Tilburg Law School Research, 8, 1-29. Mayer- Schönberger, V. y Cukier, K. (2013). The Rise of Big Data: How it's Changing the Way We Think about the World. Foreign Affairs, 92, 28-40.

Mayer- Schönberger, V. (2009). Delete. The Virtue of Forgetting in the Digital Age. Princeton: Princeton University Press.

McNealy, J. (2012). The emerging conflict between newswhorthiness and the right to be forgotten. Northern Kentucky Law Review, 39 (2), 119- 135.

Mills, J. L. (2008). Privacy: the lost right. Oxford: Oxford University Press.

Moreno, Á. y Serrano, I. (2017). El derecho al olvido digital. Especial consideración al caso chileno. En Machado, P. (coord.), Pensamiento Jurídico Central (pp. 37- 52). Valencia:Tirant lo Blanch.

Moreno, Á. (2017). Intimidad y menores. Madrid: Centro de Estudios Políticos y Constitucionales.

Muñoz, A. M. (2015). Eliminación de datos personales en internet: El reconocimiento del derecho al olvido. Revista Chilena de Derecho y Tecnología, 4 (2), 215- 261.

Pérez, A. M. (2016). Cuando google juega con la información privada... el derecho al olvido digital en Europa, una lucha de titanes. Revista La Propiedad Inmateria, 22 (julio-diciembre), 173- 186. 
Prosser, W. (1960). Privacy. California Law Review, 48 (3), 383-423.

Rallo, A. (2014). El derecho al olvido en Internet. Google versus España. Madrid: Centro de Estudios Políticos y Constitucionales.

Rosen, J. (2012). The right to be forgotten. Standford Law Review, 64, 88-92.

Rosen, J. (2000). The Unwanted Gaze the Destruction of Privacy in America (Nueva York, Random House).

Saldaña, M. N. (2011). El derecho a la privacidad en los EEUU: aproximación diacrónica a los intereses constitucionales en juego. Teoría y Realidad Constitucional, 28, 279-312.

Simón, P. (2015). El reconocimiento del derecho al olvido digital en España y en la UE. Efectos tras la sentencia del TJUE de mayo de 2014. Barcelona: Bosch.

Solove, D. (2007). The future of reputation: gossip, rumor and privacy on the internet. Yale: Yale University Press.

Solove, D. (2004). The digital person: Technology and Privacy in the Information Age. Nueva York: NYU Press.

Touriño, A. (2014). El derecho al olvido y a la intimidad en Internet. Madrid: Catarata.

Tutt, A. (2015). The revisability principle. Hastings Law Journal, 66, 1113- 1159.

Warren, S., Brandeis, L. (1890). The right to privacy, Harvard Law Review, 4 (5),193-220.

Werro, F. (2009). The right to inform v. the right to be forgotten: a transatlantic clash. Georgetown University. Center for transnational legal studies colloquium, 2, 285-300.

Westin, A. F. (1970). Privacy and Freedom. Nueva York: Atheneum.

Whitman, J. Q. (2004). The two western cultures of privacy: Dignity versus liberty. Yale Law Journal, 113, 151-1221.

Xanthoulis, N. (2013). The right to oblivion in the information age: a human- rights based approach. U.S.- China Law Review, 84- 98.

Youm, K. y Park, A. (2016). The right to be forgotten in european union law: data protection balanced with free speech? Journalism and Mass Communication Quarterly, 93 (2), 273- 295.

Zárate, S. (2013). La problemática entre el derecho al olvido y la libertad de prensa. Derecom, 13 (marzo-mayo), $1-10$. 